\title{
Elliptic integral solutions of spatial elastica of a thin straight rod bent under concentrated terminal forces
}

\author{
Giovanni Mingari Scarpello*and Daniele Ritelli ${ }^{+}$
}

\begin{abstract}
In this article we solve in closed form a system of nonlinear differential equations modelling the elastica in space of a thin, flexible, straight rod, loaded by a constant thrust at its free end. Common linearizations of strength of materials are of course not applicable any way, because we analyze great deformations, even if not so large to go off the linear elasticity range. By passing to cylindrical coordinates $\rho, \theta, z$, we earn a more tractable differential system evaluating $\rho$ as elliptic function of polar anomaly $\theta$ and also providing $z$ through elliptic integrals of I and III kind. Deformed rod's centerline is then completely described under both tensile or compressive load. Finally, the planar case comes out as a degeneracy, where the Bernoulli lemniscatic integral appears.
\end{abstract}

KEYWORD: Elliptic integrals, Spatial elastica, Nonlinear differential equations, Linear elasticity

NOTE: this is the post print version of the paper appeaered on Meccanica 41 (2006) 519-527 doi:10.1007/s11012006-9000-3

\section{A literature's background}

As early as 1691 Jakob Bernoulli proposed the problem to determine the deformed centerline (planar "elastica") of a thin, homogeneous, straight and flexible rod under forces and couples applied at its end. Whereas Galilei (Discorsi e dimostrazioni matematiche intorno a due nuove scienze, 1638) and Mariotte (Traité du mouvement des eaux et des autres corps fluides, 1686) had investigated the strength of beams, he studied the geometry of their deflection. In such a way, some time later, he established (Curvatura laminae elasticae, 1694) elastica's differential equation and met the same elliptic integral $\int\left(1-\gamma^{4}\right)^{-1 / 2} d \gamma$ of lemniscate rectification. He integrated it by series and proved the required line, sometimes referred as lintearia, to be shaped as a cross section of a horizontal flexible cylinder filled with water with free surface belonging to the line of thrust. The subject was also treated by his brother Johann at lessons XLIV (De curvatura lintei a fluido incumbente) and XLV, (Constructio curvae linteariae) of his Lectiones Mathematicae de methodo integralium ... annis 1691 \& 1692, issued in 1742. Daniel Bernoulli, Johann's son, had obtained in 1738 an integral expression of potential energy stored in a bent rod: in 1742 he guessed it would attain such a shape as to minimize the functional of squared curvature. Accordingly, Euler dealt elastica as an isoperimetric problem of Calculus of Variations, De curvis elasticis, 1744, arrived at elastica's ODE, and -mainly interested in the geometrical forms of elastic curves-identified nine forms of them. The subject attracted also C. Maclaurin, who realized the elastica had to be connected to elliptic integrals, see A treatise on fluxions, 1742, at $\S$ 927: The construction of the elastic curve, and of other figures, by the rectification of the conic sections.

In 1757 Euler wrote a paper, Sur la force des colonnes, concerning the buckling of columns again, where he approached the critical load through a simplified expression to the elastica's curvature. In 1770 Lagrange, Sur la figure des colonnes, did not limit himself to a calculation of critical loads already discussed by Euler, but went on to investigate the deflections when the load exceeds its critical value, using the exact curvature, and integrating by series his elastica's ODE. Euler went over the problem, always in the field of bending, again in 1770 and 1775: for all this historical concern, see [4], [5] and [18].

Some analytic solutions to elastic planar curves through elliptic integrals of I and II kind, can be read at [17], [39] and [14]. The link between elliptic functions and elastica was deemed so close, to induce to repre-

\footnotetext{
*Via Negroli, 620136 Milano giovannimingarieyahoo. it

${ }^{\dagger}$ Dipartimento di Matematica per le scienze economiche e sociali, Università di Bologna daniele.ritelli@unibo. it
} 
sent elliptic functions through suitable curved rubber rods (G. Greenhill: Graphical representation of the elliptic functions by means of a bent elastic beam, 1876).

For recent developments, see: [5], [3], [9], [6], [2]. Special planar problems can be seen at [11] which includes the effects of transverse deformation; in [8] a variety of loads (terminal, transverse, continuous) is tackled; whereas the effect of large loads can be seen at [19]. Unextendibility and circular shape of undeformed rod are (numerically) analyzed in [10] where the load is a uniform centrally directed force. Quite recently a new role has been found [26] to elastica in some areas of differential geometry (Willmore surfaces, pseudospherical surfaces) and in several applications, ranging from traditional ones (structural mechanics [24], engineering [25]), to biochemistry [22], and biology [23]. E. g. Kida [31] investigated the dynamics of a very thin vortex filament of infinitesimal core in an umbounded perfect fluid, using the Hama's (1962) Localized Induction Equation (LIE), which is asymptotically true for a very thin mobile vortex-filament. Passing to a stationary context, Kida gets expressions to curvature and torsion of the elastica, but even if his method seems to be fit to a 3D treatment, the (parametric) equations of Euler's elastica are obtained only in the planar case, and carelessly to rod elastic strain.

Let us come to a doubly curved elastica. The study of coiling of suboceanic cables, filamentary structures of biomolecules (like DNA) and bacterial fibers, the phenomenon of helix and reversal in climbing plants, lead today to a further motivation, but the problem is very old. It first appeared in Lagrange's Mécanique Analytique $2^{\text {nd }}$ edition (1811), even if the relevant ODEs system wasn't integrated. To J. Binet, Mémoire sur l'intégration des équations de la courbe élastique à double courbure, 1844, is due a first attempt of treating "la courbe élastique à double courbure en équilibre sous l'action de forces qui agissent à sas éxtremités seulement". Nevertheless he didn't perform any conclusive computation. In the same year, M. Wantzel, Note sur l'intégration des équations de la courbe élastique à double courbure, 1844, through an easier approach arrived at same differential system, but the integrals are vaguely formulated and not affronted. A completely different model dates to G. Kirchhoff, Über das Gleichgewicht und die Bewegung eines unendlich dünnen elastischen Stabes, 1859. Starting from Newton's second law, plus a linear constitutive relationship stress-strain, he obtained a set of nine PDEs in the variables: rod arc-length and time, describing its statics and dynamics, see [12]. He then demonstrated, under forces applied only to ends of a straight-linear rod, the relevant equilibrium equations to be identical with those describing the motion of a rigid body around a fixed point (spinning top). Three years later, Clebsch, Theorie der Elasticität fester Körper, 1862, gave formulae for the flexural and torsional couples for unstressed curved rod.

But the Kirchhoff-Clebsch theory, [7] and [1], is conceptually far from the Binet-Wantzel approach, whose equations were worked by Hermite, arriving ${ }^{1}$ at a system like (3). Unfortunately his final formulæ hold complex entities and some elliptic functions nowadays not used any more. In a 1880 tract [17], one can read the valuable article Der elastisch biegsame Faden im Raume, quite close to Wantzel's approach. Papers [22] and [7] collect about one hundred bibliographic entries about the subject. E. g. we highlight [32], whose authors obtained solutions for a straight rod under twisting. The same subject has been improved in [34] and [35] by determining when a DNA equilibrium configuration is stable in the sense that gives a local minimum to the sum of the segment's elastic energy and the potential of the acting forces.

Euler's work was gradually extended beyond the bending in order to model the effects of twist: the rods tend to buckle into spatial configurations (helices, three dimensional knots), with sudden jumps from one configuration to another. The Cosserat brothers' theory of the rods (Sur la statique de la ligne déformable, 1907; Sur la théorie des corps minces, 1908) was established to model the body as a line in space: the rod configuration is then defined by a right-handed rod-centred coordinate frame of directions specified at each arc-length $s$. Culmination of all the work done since Euler, it adds a "couple stress" (namely a torque per unit area) to traditional force per unit area, and the isotropic Cosserat version has six elastic constants, in contrast to classic elasticity holding only two (Lamé constants $\lambda$ and $G$ ). This model is useful ([36], [37], [38]) to understand a variety of phenomena such as snap-buckling, twisted cables flipping into loops, and super-coiling in DNA.

Langer and Singer treated elastica in space through three articles [27] , [28] and [29] but, in any case, rather far from the specific Wantzel-Hermite elasticity problem. In [27] elastica is a pure geometrical entity to be treated with methods of Differential Geometry, and the curves taken into account are closed and knotted in

\footnotetext{
${ }^{1}$ Just after Hermite's death,1901, the Sciences Paris Academy promoted, under government grant, the issue of famous mathematician's Collected Works under direction of his pupil E. Picard.who in the III volume added about fifty papers-relevant to different dates and journals but all- concerning elliptic functions applications, specially to Differential Geometry and Mathematical Physics. In such a way the book Sur quelques applications des fonctions elliptiques came to the light, even if never written by Hermite! It takes up pages from 266 to 418 of III volume of his Oeuvres and is divided in 51 chapters. Unfortunately Picard didn't care their crossed relationship with the originary articles. The planar elastica is at chapter 34; the skew one at chapter 35, pages 361-366, Oeuvres, III, Gauthier-Villars, 1912. The edition of all Hermite's papers (excluding tracts and lessons), completed after First World War, was of six volumes: four of articles and two of letters.
} 
$\mathbb{R}^{3}$. The authors start from a non-linear second order ODE in the curvature as a function of the arc-length: they classify and compute several knotted elasticae (orbitlike, wavelike, borderline), with the conclusive conjecture: the circle is the only stable closed elastica in $\mathbb{R}^{3}$. Paper [28] embeds the subject in a highly general geometric context looking for those regular curves -of fixed length- capable of minimizing the functional of squared curvature. The research is developed in an absolutely abstract way, considering the hyperbolic space of closed and free (when the constraint on arc-length is removed) elastic curves in an orientable 2-manifold of constant negative curvature. Last work's [29] purpose is to complement Hasimoto discovery of 1971, [33], that elastic curves can be regarded as solitons for LIE, in order to connect the Kirchhoff-Clebsch rod theory to LIE. Referring to standard Frenet frame, a special functional is considered adding total length, total torsion, and total squared curvature. Its equilibrium first variation equated to zero among all given-length configurations satisfying endpoint conditions, leads to the same nonlinear ODE involving curvature, torsion and arclength (see [27] and [28]), which can be solved in terms of elliptic functions. Finally, authors try to establish a bridge with classic Euler elastica equation: anyway this doesn't seem to be particularly clear. Article [29] is very extensive, and his aim is to improve the work of Hasimoto and Kida about the elastic curves and LIE connections: but it does not concern the specific problem of rod's elastica in space. As a consequence, the authors don't care neither the geometric characteristics, nor constraint and loading conditions, and don't even evaluate any deformation in space. In addition, their non-standard notation excludes a reader interested in continuum mechanics from seeing how the strain could be computed and analyzed in its geometrical features. It would be really too difficult to extract from [29] the necessary elements for computing 3D-elastica, or performing structural computations.

What the preceding papers leave uncovered about the practical needs of structural analyst and designer, that is done throughout our work. Starting from the Wantzel model, see [20] or [15], we develop a new solution for 3D elastica of a given loaded rod, in a clear, purely mechanical and self consisting way. We believe it might really have a practical use, since analytical solutions are scarce in this area.

\section{A 3D rod model}

Main difficulties about elastica are: its intrinsic nonlinearity due to not small displacements; geometric complexity of the unstressed configuration; constraints' effect such as isometry (non stretching); and finally boundary conditions.

The peculiarity of a thin rod when subjected to terminal forces and/or terminal couples, is that it buckles rather than breaks: our experience tells us that a compressed rod can buckle in any lateral direction. This is the strongest motivation for a spatial modelling of it.

Let us tackle a thin homogeneous rod, straight-linear in its unstressed configuration, say $\Omega P_{u} Q_{u}$; its endpoint $\Omega$ is clamped, while the other one $Q_{u}$, is free. A force $\vec{F}$ of fixed size, direction and sense is applied to it: then a stationary strain field, by equilibrium of active load $\vec{F}$, reactive force $-\vec{F}$, and reactive couple $\vec{M}$ takes place. We fix a cartesian reference frame $\Omega \hat{x} \hat{y} \hat{z}$ : origin at $\Omega$, the $\hat{z}$ axis parallel to $\vec{F}$, the $\hat{x}$ and $\hat{y}$ axes belonging to the plane for $\Omega$ orthogonal to $\hat{z}$. A sketch of elastica $\Gamma$ in space is $\Omega P Q$, see figure 1 .

The curvilinear abscissa along the 3D deflection curve $\Gamma$, is $s \geq 0$ with $s=0$ at $\Omega$, and $s>0$ at $P(s)$. We establish the assumptions:

$\mathrm{A}_{1}$ the rod is thin, initially straight, homogeneous, with uniform cross section and uniform flexural rigidity. This last is given by the product $E J$, where $E$ is the Young modulus, and $J$ the cross section second moment of inertia about a "neutral" axis normal to the plane of bending and passing through the central line;

$\mathrm{A}_{2}$ the rod is unloaded along all its external surface, and loaded by a force $\vec{F}=$ const. at its free end;

$\mathrm{A}_{3}$ transverse deformation is ignored, the rod is freely extendible, without isometry constraint, and with linear elasticity;

$\mathrm{A}_{4}$ due to rest of static equilibrium, no rod element undergoes acceleration.

Equilibrium vectorial equation, see [15], for the strained rod is:

$$
E J \frac{d}{d s}(P-\Omega) \times \frac{d^{3}}{d s^{3}}(P-\Omega)=\vec{F} \times \frac{d}{d s}(P-\Omega),
$$




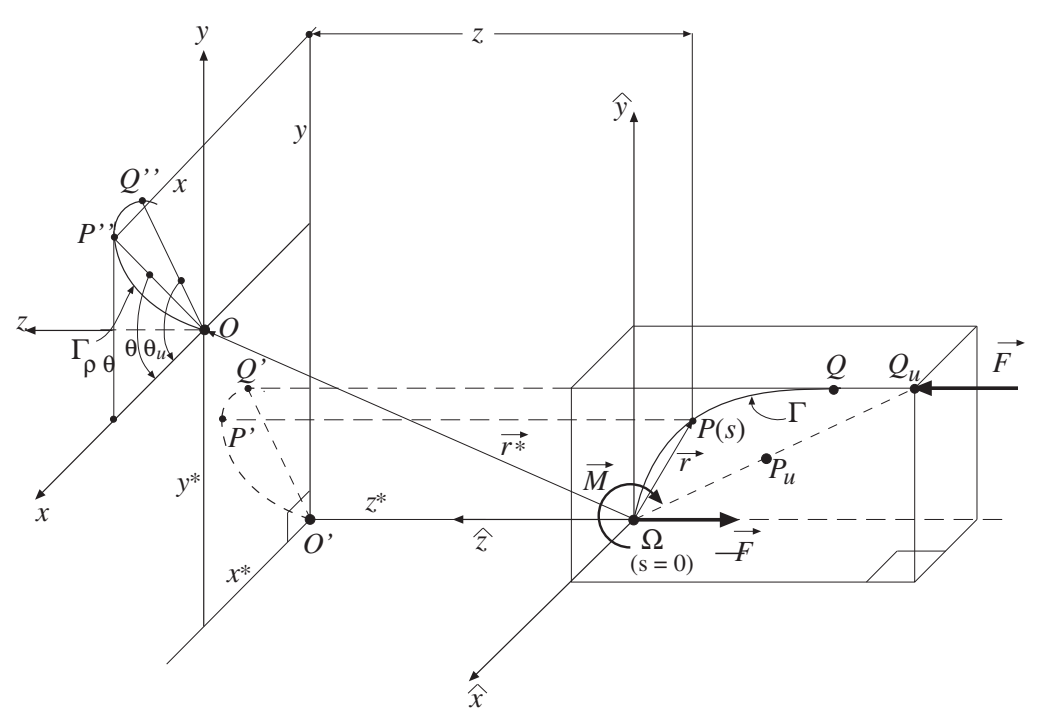

Figure 1: Elastica $\Gamma$ in space of a thin compressed rod ; $\vec{r}^{*}$ auxiliary vector to be added to $\vec{r} ; O x y z$, final threeorthogonal frame of reference; $\rho$ and $\theta$ polar coordinates superimposed upon the cartesian plane $x O y$ on which $\Gamma$ is projected as $\Gamma_{\rho \theta}$.

where $\times$ is the cross product of two vectors in $\mathbb{R}^{3}$. Putting $(P-\Omega):=\vec{r}$, after a first integration with respect to $s$, we have:

$$
E J \frac{d \vec{r}}{d s} \times \frac{d^{2} \vec{r}}{d s^{2}}=\vec{F} \times \vec{r}+\vec{V}
$$

where $\vec{V}$ is a vectorial constant (homogeneous to a moment) of integration, amenable by boundary conditions, and then assumed known. Right hand side of (1) consists of $\vec{F} \times \vec{r}$ which is $\perp$ to $\vec{F}$, plus $\vec{V}$. If we split this last as: $\vec{V}=\vec{V}_{/ / \vec{F}}+\vec{V}_{\perp \vec{F}}$, our right hand side will consist of a first term $\vec{V}_{/ / \vec{F}}$, say: $=h \vec{F}$ (where $h$ is a scalar constant), plus a second term $\vec{F} \times \vec{r}+\vec{V}_{\perp \vec{F}}$ orthogonal to $\vec{F}$ :

$$
h \vec{F}+\left(\vec{F} \times \vec{r}+\vec{V}_{\perp \vec{F}}\right) .
$$

In order to get rid of any term orthogonal to $\vec{F}$, we translate our reference frame from $\Omega \hat{x} \hat{y} \hat{z}$ to $O x y z$ with the origin moved from $\Omega$ to $O$ and the new axes parallel to the old ones. Let it be $\vec{r}^{*}$ the vector $(\Omega-O)$ to be added to $\vec{r}$ so that $\vec{F} \times\left(\vec{r}+\vec{r}^{*}\right)+h \vec{F}$ equates the right hand side of equation (1), namely: $\vec{F} \times\left(\vec{r}+\vec{r}^{*}\right)+h \vec{F}=\vec{F} \times \vec{r}+\vec{V}$. We get:

$$
\vec{F} \times \vec{r}^{*}+h \vec{F}=\vec{V},
$$

which is a vectorial equation for $h$ and the unknown constant vector $\vec{r}^{*}:=\left(x^{*}, y^{*}, z^{*}\right)$. Accordingly, the above equation is projected to $\Omega \hat{x} \hat{y} \hat{z}$ in a triple of scalar equations:

$$
\left\{\begin{array}{l}
F_{\hat{y}} z^{*}-F_{\hat{z}} y^{*}+h F_{\hat{x}}=V_{\hat{x}} \\
x^{*} F_{\hat{z}}-F_{\hat{x}} z^{*}+h F_{\hat{y}}=V_{\hat{y}} \\
F_{\hat{x}} y^{*}-F_{\hat{y}} x^{*}+h F_{\hat{z}}=V_{\hat{z}}
\end{array}\right.
$$

With $F_{\hat{x}}=F_{\hat{y}}=0, F_{\hat{z}}=F$, the solutions are:

$$
x^{*}=\frac{V_{\hat{y}}}{F}, y^{*}=-\frac{V_{\hat{x}}}{F}, h=\frac{V_{\hat{z}}}{F} .
$$

Therefore, the right hand side of equation (1) can be written as $\vec{F} \times \vec{r}^{*}+h \vec{F}$ provided that (2) holds. The third of (2) gives the $h$ value; the first two define the position of origin $O$ of new -and final- frame $O x y z$ (see figure 1 ), whose plane $x O y$ is far $z^{*}$ from the old plane $\hat{x} \Omega \hat{y}$. Because $z^{*}$ does not appear in (2), then it is arbitrary: 
as a matter of fact the component $z^{*}$ of $\vec{r}^{*}$ couldn't give any contribution to $\vec{F} \times \vec{r}^{*}$ : therefore $O$ will be placed in space unless of an arbitrary translation of size $\left|O^{\prime}-\Omega\right|=z^{*}$ along $\vec{F}$ itself. Of course the moment $\vec{V}$ could be zero and then $h=0$; alternatively $\vec{V}$ could be a non-zero planar vector with $V_{\hat{z}}=0$, and $h=0$ again: we shall see how such cases will lead to a planar elastica. With previous assumptions, referring again to 3D-sketch of figure 1, equation (1) can be written:

$$
E J \frac{d \vec{r}}{d s} \times \frac{d^{2} \vec{r}}{d s^{2}}=\vec{F} \times \vec{r}^{*}+h \vec{F} .
$$

Multiplying both sides in scalar and vectorial way by $\frac{d \vec{r}}{d s}$, one obtains, see $[15,16]$, that $O x y z$ components $x(s), y(s), z(s)$ of $\vec{r}$ shall satisfy the nonlinear differential system:

$$
\left\{\begin{array}{l}
h z^{\prime}+x y^{\prime}-y x^{\prime}=0 \\
z^{\prime \prime}+\xi\left(x x^{\prime}+y y^{\prime}\right)=0, \\
x^{\prime 2}+y^{\prime 2}+z^{\prime 2}=1
\end{array}\right.
$$

to be solved knowing four boundary values for $x, y, z, z^{\prime}$, where ' means derivative with respect to $s$. By $\xi$ we mean the ratio:

$$
\xi=\frac{F}{E J} \lesseqgtr 0,
$$

of $z$-component $F \lesseqgtr 0$ of $\vec{F}$ and flexural rigidity $E J>0$. By figure $1, \xi<0$ means tensile stress, and $\xi>0$ compression.

\section{Explicit integration}

We divide explicit integration of (3) into several steps.

\subsection{Changing to cylindrical coordinates}

System (3) consists of three coupled nonlinear scalar equations, each having at its left hand side a trinomial structure: then any approach for separating variables is not quick. One way to deal with such equations, is to pass from cartesian $x(s), y(s), z(s)$, to cylindrical coordinates $\rho(s), \theta(s), z(s)$ :

$$
\left\{\begin{array}{l}
x(s)=\rho(s) \cos \theta(s), \\
y(s)=\rho(s) \sin \theta(s), \\
z(s)=z(s) .
\end{array}\right.
$$

After some handling, (3) becomes:

$$
\left\{\begin{array}{l}
\rho^{2} \frac{d \theta}{d s}+h \frac{d z}{d s}=0, \\
\frac{d^{2} z}{d s^{2}}+\xi \rho \frac{d \rho}{d s}=0, \\
\left(\frac{d \rho}{d s}\right)^{2}+\rho^{2}\left(\frac{d \theta}{d s}\right)^{2}+\left(\frac{d z}{d s}\right)^{2}=1,
\end{array}\right.
$$

so that first and second equation lose their trinomial form. We will solve the system with the following values at the clamped boundary, namely $s=0$, see figure 1 :

$$
\left\{\begin{array}{l}
\rho(0)=0 \\
\theta(0)=\theta_{u} \\
z(0)=z^{*} \\
z^{\prime}(0)=0
\end{array}\right.
$$

Second equation is that of the plane which the unstrained rod is belonging to. By last one, clamped end does not undergo any flexional rotation. Integrating the second of (4), we get:

$$
\frac{d z}{d s}=-\frac{1}{2} \xi \rho^{2}+C,
$$


which, applying above boundary conditions, gives $C=0$ : then $z$ grows (decreases) with $s$ under tensile (compressive) load, as it shall be.

\section{$3.2 \theta$-solution}

Putting last equation in first of (4), it can be soon integrated:

$$
\theta(s)=\theta_{u}+\frac{h}{2} \xi s .
$$

Therefore the "torsion angle", locally characterizing any double curvature line, and not to be confused with torsional strain due to a torque, is for our elastica growing proportionally to $s$ : so that their points increasingly far from the clamped end, are more deviated from planarity. Check: without any force, $\xi=0$, no deviation from the plane $\theta=\theta_{u}$ will occur. But under load, $\xi \neq 0$, skewness of elastica is depending upon having a nonzero $h$.

\section{$3.3 \rho$-equation and elliptic functions}

Inserting $C=0$, and expressions to $d z / d s$ and $\theta(s)$ in third of (4), we get $\rho$ - differential equation:

$$
\pm d s=\frac{d \rho}{\sqrt{1-\frac{1}{4} h^{2} \xi^{2} \rho^{2}-\frac{1}{4} \xi^{2} \rho^{4}}},
$$

which shows that if $\xi=0, d s=d \rho$, namely: planar elastica is the same straight line of unstressed rod. By (7) we see $\xi$ cannot be infinite -not only for physical obvious causes- in order to keep the square root real. Furthermore for opposite $\xi$ values, equation (7) is invariant, but we will see some difference not before long. Putting in (7)

$$
\psi(h, \xi)=\sqrt{h^{4}+\frac{16}{\xi^{2}}}, 0<A^{2}:=\frac{\psi-h^{2}}{2}<\frac{\psi+h^{2}}{2}:=B^{2},
$$

then, choosing the plus sign because $s$ increases with $\rho$, we have:

$$
\frac{|\xi|}{2} s(\rho)=\int_{0}^{\rho} \frac{d r}{\sqrt{\left(B^{2}+r^{2}\right)\left(A^{2}-r^{2}\right)}} .
$$

Such a equation:

i) provides arc-length $s=s(\rho)$ of deformed rod once and for all, after static and elastic equilibria have been achieved;

ii) holds assumption $\rho(0)=0$;

iii) implies $0 \leq \rho<A$, a condition necessary to the square root's reality.

By formula 214.00, page 50 of [13], if:

$$
\sin \psi=\sqrt{\frac{\rho^{2}\left(A^{2}+B^{2}\right)}{A^{2}\left(\rho^{2}+B^{2}\right)}} ; k=\frac{A}{\sqrt{A^{2}+B^{2}}} ; g=\frac{1}{\sqrt{A^{2}+B^{2}}},
$$

then:

$$
\sin \psi=\operatorname{sn}\left(\frac{|\xi|}{2 g} s, k\right),
$$

where $\operatorname{sn}(\varphi, k)$ is Jacobi sine amplitude of argument $\varphi$ and modulus $0 \leq k \leq 1$. Equating the above $\sin \psi$ expressions and solving for $\rho(s)$, one gets radial solution:

$$
\rho(s)=\frac{A B \operatorname{sn}\left(\frac{|\xi|}{2 g} s, k\right)}{\sqrt{B^{2}+A^{2} \mathrm{cn}^{2}\left(\frac{|\xi|}{2 g} s, k\right)}},
$$


where $\mathrm{cn}(\varphi, k)$ is Jacobi cosine amplitude. Then $\rho$-coordinate will grow with $s$, till to $A$. Choosing afterwards minus sign in (7) for $d \rho / d s, \rho$ will go down to zero, and so on; and a periodic behaviour will take place. Going back to our originary notation, we get:

$$
\rho(s)=\frac{2 \sqrt{2}}{|\xi|} \sqrt{\frac{1-\mathrm{cn}^{2}\left(\frac{|\xi|}{2 g} s, k\right)}{\left(\psi+h^{2}\right)+\left(\psi-h^{2}\right) \mathrm{cn}^{2}\left(\frac{|\xi|}{2 g} s, k\right)}},
$$

where:

$$
\begin{aligned}
& \frac{1}{k}=\frac{1}{k(h, \xi, \psi)}=\frac{\sqrt{2 \psi}}{\sqrt{\psi-h^{2}}}, \\
& g=g(\psi)=\frac{1}{\sqrt{\psi}},
\end{aligned}
$$

and of course $\psi$ is given by (8).

\subsubsection{Main features of elastica's planar projection $\Gamma_{\rho \theta}$}

Let us project the skew line $\Gamma$ on plane $x O y$. Then, after translating the origin from $O^{\prime}$ to $O$ by means of vector $\left(x^{*}, y^{*}\right)$, one obtains a curve $O P^{\prime \prime} Q^{\prime \prime}$, say $\Gamma_{\rho \theta}$, of polar equation:

$$
\rho(\theta)=\frac{2 \sqrt{2}}{|\tilde{\zeta}|} \sqrt{\frac{1-\mathrm{cn}^{2}\left(\frac{\theta-\theta_{u}}{g h}, k\right)}{\left(\psi+h^{2}\right)+\left(\psi-h^{2}\right) \mathrm{cn}^{2}\left(\frac{\theta-\theta_{u}}{g h}, k\right)} .}
$$

Being (12) of kind $\rho=f(\cos (\theta))$, the curve, by [21], should be a leaf, even if we have the cosine amplitude and not the circular cosine. Nevertheless we keep the qualitative information of being faced with something like a petalled flower.

By inspection, $g$ doesn't change for opposite $\xi$ values. Consequently the argument $\theta /(g h)$ of cosine amplitude is an even function of $\xi$ too. Then planar projection's shape of elastica is doubly affected by the load $\xi$ : first by (12), and also for being $k$ a function (10) of $\xi$. The elliptic function $\rho$ of the variable $\theta /(g h)$ has a period which is half of the cn one, $P=2 \mathbf{K}(k)$, being $0<k(h, \xi) \leq 1 / \sqrt{2}$. Let $N$ be the number of complete revolutions around $O$ after which the $\Gamma_{\rho \theta}$ polar pattern will repeat itself:

$$
N=\frac{P}{2 \pi}=\frac{\mathbf{K}(k)}{\pi} \geq \frac{1}{2},
$$

provided that rod succeeds to be long enough. $N$ cannot be integer, because the upper bound on $k(h, \xi)$ implies that:

$$
\frac{1}{2} \leq N=\frac{\mathbf{K}(k)}{\pi} \leq \frac{1}{\pi} \mathbf{K}\left(\frac{1}{\sqrt{2}}\right) \approx 0.5901704 .
$$

If $N$ is rational, say $N=a / b, a, b \in \mathbb{N}$, then $b P=2 \pi a$, and after $a$ complete $\theta$-turns, the function $\rho(\theta /(g h))$ will have taken for $b$ times the same values again. If $N$ is irrational, $P$ will be not rationally congruent to $\pi$, and the path will not close upon itself after a finite number of lobes. At figure 2 the case $\xi=1 / 5, h=1, \theta_{u}=$ $0,0 \leq \theta \leq 2 \pi$ has been plotted. Notice that, taking for $\xi$ the opposite value, $k$ will be different and another pattern will be obtained; in any case the graphics will not be superimposed.

\subsection{Final stage: $z$-integration}

Let us see from (4) how $\Gamma^{\prime}$ s $z$-coordinate can now be computed. Formula (5), after a first integration gives:

$$
z(s)=z^{*}-\frac{1}{2} \xi \int_{0}^{s} \rho^{2}(\zeta) d \zeta .
$$

But we have:

$$
\frac{B^{2}}{A^{2}+B^{2}}=1-k^{2}=k^{\prime 2}, \frac{A^{2} B^{2}}{A^{2}+B^{2}}=\frac{k^{2} k^{\prime 2}}{g^{2}}, \frac{\xi}{2}=\alpha,
$$




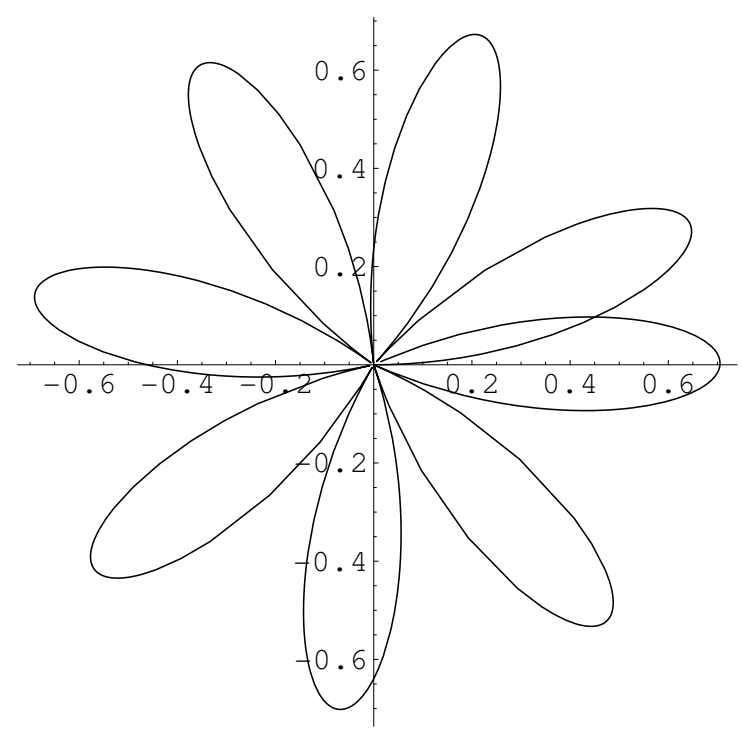

Figure 2: Planar pattern $(\rho, \theta), 0 \leq \theta \leq 2 \pi$, of skew elastica: $\xi=1 / 5, h=1, \theta_{u}=0$.

and then, inserting $\rho(s)$ :

$$
z(s)=z^{*}-\frac{1}{2} \xi \frac{k^{2} k^{\prime 2}}{g^{2}} \int_{0}^{s} \frac{\mathrm{sn}^{2}\left(\frac{\alpha}{g} \zeta, k\right)}{1-k^{2} \mathrm{sn}^{2}\left(\frac{\alpha}{g} \zeta, k\right)} d \zeta .
$$

The integral can be solved with the help of formula 337. 01, page 201 of [13]. We get:

$$
z(s)=z^{*}-\xi \frac{k^{2}}{2 \alpha g}\left[\Pi\left(\frac{\alpha}{g} s, k^{2}, k\right)-F\left(\frac{\alpha}{g} s, k\right)\right],
$$

$\Pi\left(\varphi, p^{2}, k\right)$ and $F(\varphi, k)$ are the Legendre incomplete elliptic integrals of III and I kind, with $\varphi, p^{2}, k$ amplitude, parameter and modulus, respectively.

Formulæ (6), (9), (14) for $\theta(s), \rho(s), z(s)$ give a full parametric description of our 3D-elastica of a thin equilibrium rod under terminal loads, knowing: applied forces, material Young modulus, and cross section dimensions. The parameter is the arc-length $s \geq 0$ counted from $\Omega$.

Notice that $\rho(s)$ is obtained through the Jacobi elliptic function cosine amplitude, whereas $z(s)$ through Legendre incomplete elliptic integrals of I and III kind.

\section{A meaningful degeneracy, $h=0$ : planar strain}

In order to model elastica in space under a load $\vec{F}$, we gave to the constant $\vec{V}$ the structure $h \vec{F}=\vec{V}$. All the preceding treatment has then accordingly developed with $h>0$.

A special degeneracy arises when the vector $\vec{V}$ has the $\hat{z}$-component zero. In such a case, by (2), we have $h=0$, namely, see (6), elastica belongs to a plane $\theta=\theta_{u}$. By (7), putting $h=0$ and $\rho \sqrt{|\xi| / 2}=\gamma$, we get:

$$
s=\frac{2}{\sqrt{|\xi|}} \int_{0}^{\rho \sqrt{|\xi| / 2}} \frac{d \gamma}{\sqrt{1-\gamma^{4}}}
$$

which is the Jakob Bernoulli lemniscatic integral, $|\gamma|<1$. Accordingly, putting $\gamma=\cos \varphi$, it is easy reduced to Legendre form:

$$
s \sqrt{\frac{|\xi|}{2}}=\int_{0}^{\pi / 2} \frac{d \varphi}{\sqrt{1-\frac{1}{2} \sin ^{2} \varphi}}-\int_{0}^{\arccos (\rho \sqrt{|\xi| / 2})} \frac{d \varphi}{\sqrt{1-\frac{1}{2} \sin ^{2} \varphi}} .
$$


Or :

$$
\rho(s)=\sqrt{\frac{2}{|\xi|}} \text { cn }\left[s \sqrt{\frac{|\xi|}{2}}-\mathbf{K}\left(\frac{1}{\sqrt{2}}\right)\right],
$$

which provides the radial equation to polar parametric representation of planar elastica laid upon plane $\theta=\theta_{u}$, $\mathrm{K}$ being the complete elliptic integral of I kind. Let us pass to that providing $z$. By (13), and [13] formula 312.03 page 193, recalling that elliptic integrals of I and II kind are odd functions of their amplitude $\varphi$, we get:

$$
\begin{aligned}
z(s)= & z^{*}-\sqrt{\frac{|\xi|}{2}}\left\{2 E\left[s \sqrt{\frac{|\xi|}{2}}-\mathbf{K}\left(\frac{1}{\sqrt{2}}\right)\right]-F\left[s \sqrt{\frac{|\xi|}{2}}-\mathbf{K}\left(\frac{1}{\sqrt{2}}\right)\right]+\right. \\
& \left.+2 E\left[\mathbf{K}\left(\frac{1}{\sqrt{2}}\right)\right]+F\left[\mathbf{K}\left(\frac{1}{\sqrt{2}}\right)\right]\right\},
\end{aligned}
$$

being $E(\varphi, k)$ the Legendre incomplete elliptic integrals of II kind, where the value $1 / \sqrt{2}$ of modulus in expressions for $\mathrm{cn}, F, E$, has been omitted.

Our elastica is then always laid upon the same plane $\theta=\theta_{u}$ defined through the rod's original unstrained direction and the straightlines of $\vec{F},-\vec{F}$. Above formulae for $\rho(s)$ and $z(s)$ are then providing the "natural" equations of the elastica through the parameter $s \geq 0$ counted from $\Omega$.

\section{Conclusions}

We construct exact analytic solutions to non linear ODEs (4) describing large deflections in space of a straight, prismatic, flexible rod bent under terminal active thrust of whichever direction coplanar to the rod straightline. We face with great deformations, but not so large to go off limits of linear elasticity: nevertheless the analytical problem succeeds in being highly nonlinear. Originary discouraging equations have been reduced to a more tractable form, by passing from $\Omega \hat{x} \hat{y} \hat{z}$ to $O x y z$ and after, from $O x y z$ to cylindrical coordinates. Main achievements:

1. the torsional angle $\theta$ of the double curvature elastica $\Gamma$, is soon found as proportional to arc-length $s$;

2. the planar radius $\rho$ on the polar reference upon $O x y$ is an elliptic, and then periodic, function of arc-length $s$, with upper bound $A$ :

$$
A(h, \xi)=\sqrt{\frac{1}{2 \xi}\left(\sqrt{16+h^{4} \xi^{2}}-h^{2} \xi\right)} ;
$$

3. $z=z(s)$ is computed by a formula where elliptic integrals of both III and I kind appear;

4. $\rho$ and $z$ can also be obtained as functions of polar anomaly $\theta$;

5. the alternative: compression/tension, namely $\xi>0$ or $\xi<0$, on the elastica's shape, is solely impacting the $k$ modulus (10), which is conditioning both $\rho(\theta)$ and $z(s)$;

6. elastica's planar projection $\Gamma_{\rho \theta}$ of equation $\rho=f(\theta)$, shows a geometric pattern which will repeat itself, around $A$, periodically after $N$ turns: $1 / 2 \leq N \leq(1 / \pi) \mathbf{K}(1 / \sqrt{2})$, whenever $N$ is rational. If not, the $\Gamma_{\rho \theta}$ path will not close upon itself;

7. the special case $h=0$ gives back the same lemniscatic integral discovered by Jakob Bernoulli in 1694 just while he was working about planar elastica. In addition, $z=z(s)$ is given by a formula with elliptic integrals of II and I kind, and not III and I any more.

Let us close with a motivational note: recent aerospace, offshore and long span constructions which have been or are being manufactured, should challenge the researchers to try to develop nonlinear analyses to the same degree of simplicity of computation like those established for the linear ones. 


\section{Acknowledgements}

The authors are indebted to their friend Aldo Scimone who drew the first figure of this paper: they hereby take opportunity for thanking him warmly.

Research supported by MIUR grant: Equazioni differenziali e problemi geometrici

\section{References}

[1] Dill E. H., 'Kirchhoff's theory of rods', Arch. History of exact sciences, 44 (1992), 1-23.

[2] Panayotounakos D. E., Theocaris P. S., 'Exact solution for an approximate differential equation of a straight bar under conditions of non-linear equilibrium', Int. J. Non-linear Mechanics 21 (1986), 421-429.

[3] Chucheepsakul S., Phungpaigram B., 'Elliptic integral solutions of variable -arc-length elastica under an inclined follower force', Z. Angew. Math. Mech., 84 (2004), 29-38.

[4] Fraser C. G., 'Euler's investigation of the elastica', Centaurus, 34 (1991), 211-246.

[5] Basoco M. A., 'On the inflexional elastica', Am. Math. Monthly, 48 (1941), 303-309.

[6] Panayotounakos D.E., Theocaris P.S., 'Exact solution of the non-linear differential equation concerning the elastic line of a straight rod due to terminal loading', Int. J. Non-linear Mechanics, 17 (1982), 395- 402.

[7] da Fonseca A. F. , de Aguiar M. A. M., 'Solving the boundary value problem for finite Kirchhoff rods', Physica D, 181 (2003), 53- 69.

[8] Panayotounakos D. E., Theocaris P.S., 'Analytic solutions for nonlinear differential equations describing the elastica of straight bars: theory', Journal of the Franklin Institute, 325 (1988), 621-633.

[9] Goto Y., Yoshimitsu T., Obata M., 'Elliptic integral solutions of plane elastica with axial and shear deformations', Int. J. Non-Linear Mechanics, 26 (1990), 375-390.

[10] Dickey R. W. , Rosemann J. J., 'Equilibria of the circular elastica under a uniform central force field', Quart. of Appl. Mathematics, 51 (1993), 201-216.

[11] Sotiropoulu A. B., Panayotounakos D. E., 'Exact parametric analytic solution of the elastica ODEs for bars including effects of the transverse deformation', Int. J. Non-Linear Mechanics, 39 (2004), 1555-1570.

[12] Love A. E. H., A treatise on the mathematical theory of elasticity $4^{\text {th }}$ ed. 1927, reprint Dover, New York, 1944, pp. 1-32.

[13] Byrd P. F., Friedman M. D., Handbook of elliptic integrals for engineers and scientists, $2^{\text {nd }}$ ed., Springer Verlag, New York, 1971.

[14] Tricomi F. G. , Funzioni ellittiche, Zanichelli, Bologna, 1951.

[15] Landau L., Lifchitz E. , Théorie de l'élasticité, MIR, Moscow 1967.

[16] Cercignani C., Spazio, tempo, movimento, Zanichelli, Bologna 1991.

[17] Schell W. , Theorie der Bewegung und der Kräfte, II, B. G. Teubner Druck u. Verlag, Leipzig, 1880.

[18] Benvenuto E., La scienza delle costruzioni e il suo sviluppo storico, Sansoni, Firenze 1981.

[19] Lardner T.J., 'A note on the elastica with large loads', Int. J. solids structures, 21 (1985), 21-26.

[20] Appell P. , Lacour E., Fonctions elliptiques, Gauthier-Villars, Paris 1922.

[21] Loria G., Curve piane speciali, I, Zanichelli, Bologna, 1930.

[22] Matsutani, S. , 'Statistical mechanics of elastica on a plane: origin of the MKdV hierarchy', J. Phys. A., 31, 11 (1998), 2705-2725. 
[23] Stump D.N., Fraser W. B., 'Multiple solutions for writhed rods: implications for DNA supercooling', Proc. R. Soc. Lond. A, 456 (2000), 455-467.

[24] Golley B.W., 'Large deflections of bars bent through frictionless supports', Int. J. Non-Linear Mechanics 19 (1984), 1-9.

[25] Sun Ming-Gang, 'Behaviour of a post-buckling riser vibrating due to effects of waves and current', Int. J. Non-Linear Mechanics, 27 (1992), 437-445.

[26] Perline R., 'The role of elastica in geometry', in Wojcik, Daniel et al. (Eds), Proceedings of the first nonorthodox school on nonlinearity and geometry, Luigi Bianchi days, Warsaw, September 21-28, 1995 Polish Scientific Publishers PWN, Warsaw, 1998, 359-364.

[27] Langer J., Singer D.A., 'The total squared curvature of closed curves', J. Differential Geometry, 20 (1984), $1-22$.

[28] Langer J., Singer D.A., 'Knotted elastic curves in $\mathbb{R}^{3}$, J. London Math. Soc. , 2, 30 (1984), 512-520.

[29] Langer J., Singer D.A., 'Lagrangian aspects of the Kirchhoff elastic rod', SIAM Review, 38, 4 (1996), 605-618.

[30] Shi, Y., Hearst J. E. , 'The Kirchhoff elastic rod, the nonlinear Schrödinger equation, and DNA supercooling', J. Chem. Phys., 101, 6 (1994), 5186-5200.

[31] Kida, S., 'A vortex filament moving without change of form', J. Fluid Mechanics, 112 (1981), 397-409.

[32] Tobias I., Coleman B. D. , Olson, W. K. , 'The dependence of DNA tertiary structure on end conditions: Theory and applications for topological transitions', J. Chem. Phys., 101, 12 (1994), 10990.

[33] Hasimoto, H., 'Motion of a vortex filament and its relation to elastica', J. Phys. Soc. Japan, 31 (1971), 293-294.

[34] Irwin T. , Swigon D., Coleman B. D. : 'Elastic stability of DNA configurations. I. General theory', Physical Review E, 61 1, (2000), 747-758.

[35] Coleman B. D., Swigon D., Irwin T., 'Elastic stability of DNA configurations. II. Supercoiled plasmids with self contact', Physical Review E, 61 1, (2000), 759-770.

[36] Lakes R., 'Experimental methods for study of Cosserat elastic solids and other generalized elastic continua' in: Continuum models for materials with micro-structure, Mühlhaus, H. (Ed.) J. Wiley, New York, 1995, pp. 1-22.

[37] Antman S., Nonlinear problems of elasticity, Springer-Verlag, New York, 1995.

[38] van der Heijden G. H. M. , 'The static deformation of a twisted elastic rod constrained to lie on a cylinder', Proc. R. Soc. Lond. A , 457, (2001), 695-715.

[39] Burgatti, P. , Teoria matematica dell'elasticità, Zanichelli, Bologna, 1931. 